\title{
Prognostic significance of transient ST segment changes after coronary artery bypass surgery: a long-term (4-10 year) follow up study
}

Deven Patel, David Mulcahy, Nicholas Curzen, Anne Sullivan, David Cunningham, Jane Sparrow, Christine Wright, Arshed Quyyumi, Kim Fox

\begin{abstract}
Objective-To assess the long-term (four to 10 years) prognostic significance of transient ST segment changes on ambulatory ST segment monitoring after coronary artery bypass grafting (CABG).

Patients and methods-76 patients (67 men, nine women) underwent CABG between 1982 and $1984(n=31)$ and between 1987 and $1988(n=45)$ and at a mean age of 57. All underwent 48 hours of ambulatory ST segment monitoring at a mean of 19 weeks after surgery. The results were available for assessment. All general practitioners were contacted and patients' notes reviewed. Patients were contacted by telephone. Details were recorded of intervening events (acute myocardial infarction, unstable angina, need for further revascularisation, and deaths). Event free survival curves were produced for those with and without transient ST segment changes during routine postoperative ambulatory ST segment monitoring.
\end{abstract}

Results-During 3213 hours of monitoring after CABG, 21 (27.6\%) of 76 patients had transient ST segment changes, of which $70 \%$ were silent. Over a mean 70 month follow up period, patients with such ischaemic changes were no more likely to have either an objective (myocardial infarction or cardiac death) or subjective (unstable angina or another revascularisation) event than those patients without ischaemic changes. This finding was the same in patients operated on between 1987 and 1988 and between 1982 and 1984. Conchusions-Although ambulatory ST segment monitoring is becoming increasingly popular in some countries as a routine investigation for ischaemia in various coronary subgroups, the findings of such an investigation, when performed after CABG, do not help to identify a subgroup more likely to have an adverse outcome during up to 10 years of follow up. There seems to be no reason to perform this investigation after surgery, and particularly to refer patients for reinvestigation because of the detection of predominantly silent ST segment changes of uncertain relevance.

(Br Heart f 1993;70:337-341)
It has been clearly shown that transient, and predominantly silent, episodes of ST segment depression occur commonly during daily life in patients with stable coronary artery disease, ${ }^{12}$ unstable angina, ${ }^{34}$ and at various phases after acute myocardial infarction. ${ }^{56}$ Many authors have reported that such transient ischaemic episodes are associated with an adverse short and medium term prognosis in patients with stable angina, ${ }^{78}$ unstable angina, ${ }^{34}$ and after infarction. ${ }^{56}$ Other authors have not concurred with these findings in patients with stable angina. ${ }^{9}$ There is considerable variation in what is reported as an end point in the various studies; some authors included subjective end points such as the need for revascularisation, whereas others assess the objective end points of acute myocardial infarction and sudden cardiac death separately.

Whereas it was initially reported that after coronary artery bypass grafting (CABG) the presence of transient and almost exclusively silent ischaemic ST segment changes during daily life might be associated with an adverse short term prognosis, ${ }^{10}$ another longer term follow up study reported that the presence of such ischaemic changes in the stable phase after surgery was not of prognostic relevance. ${ }^{11}$

We have assessed the long term (up to 10 years) prognostic importance of transient ST segment changes during daily activities in 76 patients who had CABG between 1982 and 1988 and who subsequently underwent routine ambulatory ST segment monitoring. These patients have been previously reported as part of studies assessing the effects of CABG on such transient ST segment changes detected by ambulatory monitoring. ${ }^{1213}$

\section{Patients and methods}

Seventy six patients ( 67 men, nine women) of mean age 57 (range 40-75) years underwent CABG between January 1982 and November 1988. The results of subsequent routine monitoring of ambulatory ST segment are available. Almost all patients had been operated on because of severe angina. Fifteen patients had left main coronary artery disease, 47 had three vessel, 11 two vessel, and three single vessel disease. Therefore, 62 patients $(81 \%)$ had three vessel or left main coronary 
artery disease. During surgery the mean number of bypass grafts (including the internal mammary artery graft) was 3.3 (range 1-5). No patient had developed significant conduction disturbances likely to effect the interpretation of the ST segment after surgery.

\section{AMBULATORY ST SEGMENT MONITORING}

Forty eight hours of ambulatory ST segment monitoring was performed with pre-gelled electrodes to record two bipolar leads, anterior lead CM5 and a modified inferior lead. Sites and methods of application have been previously described. ${ }^{14}$ Two channel recordings were then obtained on magnetic tape by a frequency modulated dual channel recorder (Oxford Medilog 2, frequency response $0.05-40 \mathrm{~Hz}$ ). The frequency response of this system is sufficient to record and display the ST segment accurately. Tapes were then analysed at 60 to 120 times normal speed with an Oxford Medilog MA20 scanner, and all printouts were at $25 \mathrm{~mm} / \mathrm{s}$. Significant ST segment depression was defined as planar or downsloping ST segment shift of $>1 \mathrm{~mm}$ measured $0.08 \mathrm{~s}$ after the $\mathrm{J}$ point that persisted for $>1 \mathrm{~min}$. Changes in the $T$ wave were not regarded as evidence of myocardial ischaemia unless they were accompanied by significant ST segment changes.

During the period of ambulatory monitoring, each patient kept a detailed angina diary to record each episode of pain, the activity at the onset of symptoms, and the requirement for glyceryl trinitrate. They pressed an event marker at the onset of symptoms, which marked the magnetic tape allowing for automatic display of the electrocardiogram at the time of playback. All patients were encouraged to continue their normal daily activities during monitoring.

\section{MEDICATIONS DURING MONITORING}

Of the 76 patients undergoing routine ambulatory ST segment monitoring after CABG $59(78 \%)$ had no antianginal treatment.

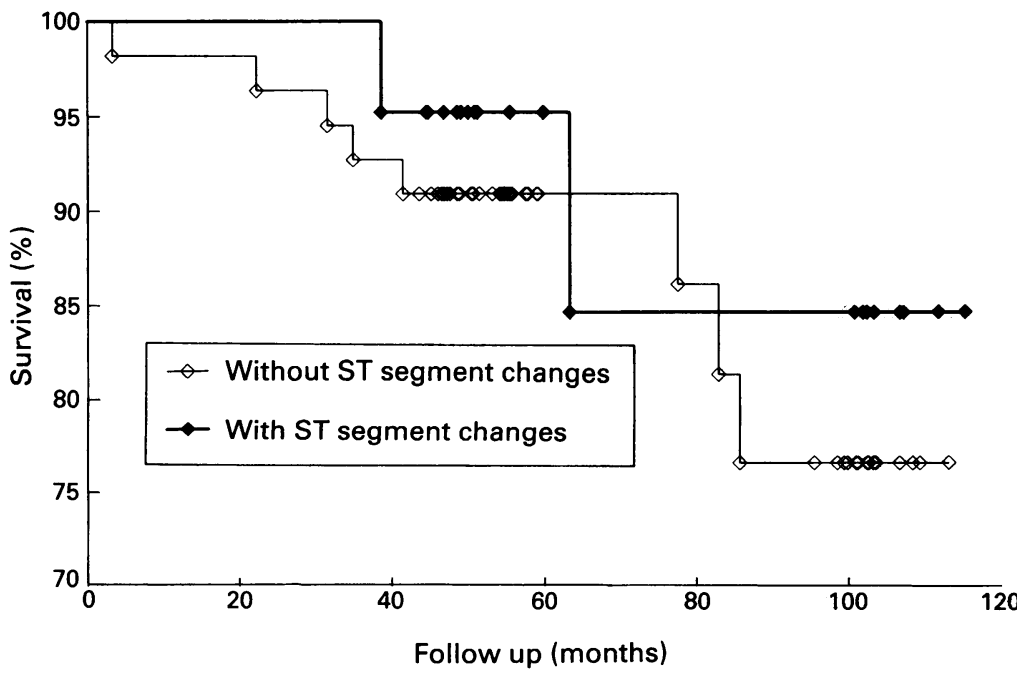

Figure 1 Cumulative objective (acute myocardial infarction and cardiac death) even free survival over a mean 70 month follow up period in 76 patients with or without transient $S T$ segment changes on routine ambulatory $S T$ segment monitoring after $C A B G$.
Seventeen (22\%) patients had antianginal treatment; in eight $(47 \%)$ of these cases the treatment was for hypertension.

\section{FOLLOW UP OF PATIENTS}

The notes of all patients were reviewed and the general practitioner contacted to establish whether the patient was alive. Surviving patients were contacted and questioned about (a) hospital admissions after CABG, (b) the need for further intervention, (c) symptoms of angina and when it developed after CABG, (d) medication taken. Cause of deaths was sought from the general practitioner or from the relevant hospital. Objective end points recorded were acute myocardial infarction and cardiac death. Subjective end points included admission to hospital with unstable angina and need for further revascularisation. Non-cardiac deaths, were also recorded.

\section{STATISTICAL ANALYSIS}

We constructed Kaplan-Meier cumulative survival curves in the conventional way, with objective and a combination of objective and subjective end points. Potential differences between survival curves were analysed with the log rank test.

\section{Results \\ AMBULATORY ST SEGMENT MONITORING AFTER CABG}

The 76 patients underwent a total of 3213 hours of ambulatory ST segment monitoring (mean 42.3 hours) at a mean of 19 (range 2-78) weeks after CABG. Twenty one (28\%) patients had a total of 84 transient episodes of ST segment change during monitoring, of which $25(30 \%)$ were painful. Eighteen of 59 (31\%) patients without antianginal treatment had ST segment changes, as did three of 17 patients $(18 \%)$ with treatment. The mean number of grafts received was 3.0 in those with ST segment changes during monitoring, and 3.4 in those without. In no case had the findings of ambulatory ST segment monitoring after CABG altered clinical management, as the relevance of such findings was not known.

EVENTS RECORDED DURING FOLLOW UP AFTER AMBULATORY MONITORING

Among the 76 patients there were seven deaths, of which six were cardiac in origin. Five patients had non-fatal acute myocardial infarction. There were eight reported admissions with unstable angina, and one patient underwent further revascularisation. Symptoms of angina recurred in a total of 30 patients at a mean of $\mathbf{4 3}$ months follow up.

RELATION BETWEEN RESULTS OF AMBULATORY ST SEGMENT MONITORING AND END POINTS Figure 1 shows cumulative event free survival for the study group in terms of acute myocardial infarction or cardiac death over a mean of 70 months (range 22-115) follow up after monitoring, and figure 2 assesses outcome with acute myocardial infarction, cardiac 


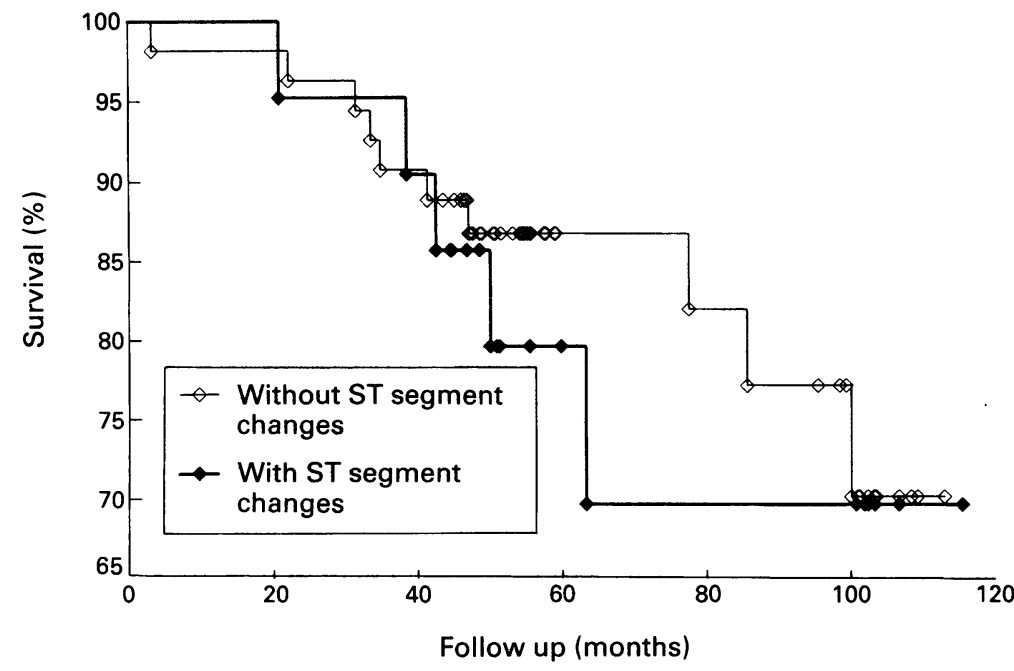

Figure 2 Cumulative total (acute myocardial infarction, cardiac death, unstable angina, and further revascularisation) event free survival in 76 patients with or without transient $S T$ segment changes on routine ambulatory ST segment monitoring after $C A B G$.

death, unstable angina, and further revascularisation as end points. The presence of transient ST segment changes during routine monitoring does not help to identify a subgroup more likely to have an adverse outcome after CABG.

Figure 3 assesses the relation between the presence or absence of ST segment changes on ambulatory monitoring and subsequent acute myocardial infarction or cardiac death in 31 patients operated on between 1982 and 1984 and who were followed up for eight to 10 years and in 45 patients operated on between 1987 and 1988 who were followed up for four to five years. The presence of transient ST segment changes after CABG are of little predictive value in identifying those more likely to have myocardial infarction or cardiac death in either the intermediate or long term after CABG.

Nine patients had one or more episodes of ST segment change associated with pain during monitoring. These patients were not more likely than other patients to have an objective

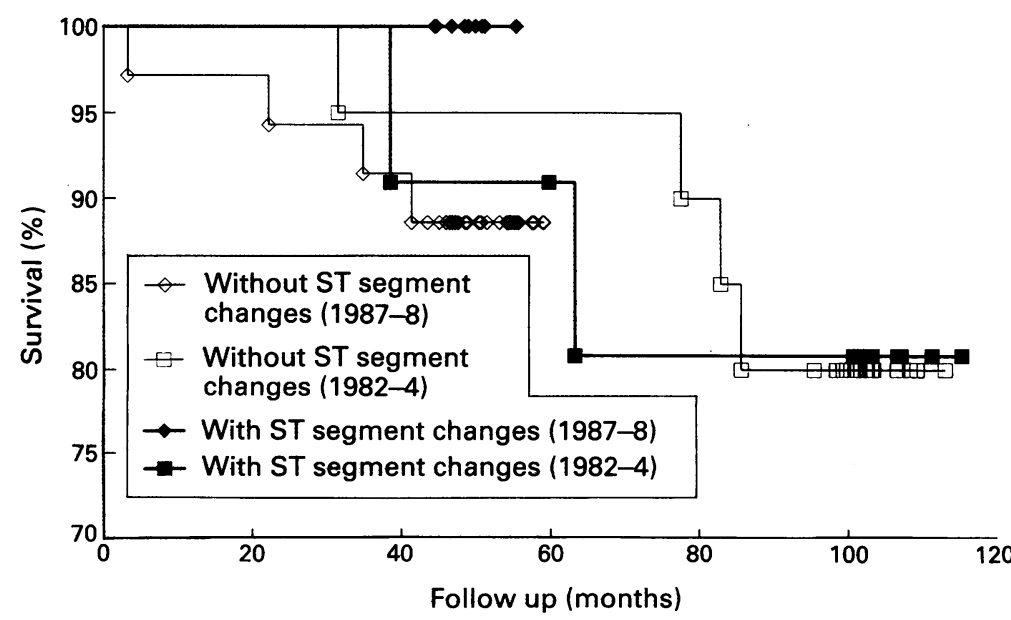

Figure 3 Cumulative objective (acute myocardial infarction, cardiac death) event free survival in 31 patients with or without transient ST segment changes on ambulatory $S T$ segment monitoring who underwent CABG between 1982 and 1984, and 45 patients treated similarly between 1987 and 1988 cardiac event over a mean 70 month follow up period. There was no increased frequency of objective cardiac events in patients without antianginal treatment compared with those on such treatment either for recurrent angina or for hypertension.

\section{Discussion}

Although ambulatory ST segment monitoring has been used extensively in cardiovascular research over the past 15 years, it is not yet widely used in routine clinical investigations in patients with various coronary syndromes. Although there is some conflict as to whether transient ST segment changes during daily life in patients with stable angina indicates an adverse prognosis, ${ }^{7-9}$ the presence of such changes in patients with unstable angina pectoris seems to be associated with an adverse short-term outcome, ${ }^{34}$ and continuous ST segment monitoring in the coronary care unit may help to identify those patients more likely to benefit from early investigation and intervention. Recent evidence also suggests that such ST segment changes are associated with an adverse outcome in patients undergoing non-cardiac surgery, ${ }^{15} 16$ but these findings may simply suggest that people with transient ischaemia during daily life are more likely to have coronary artery disease than those without. It is not entirely surprising that those with significant coronary artery disease are more likely to have perioperative cardiac events than those without objective evidence of coronary disease. It has been reported that transient ST segment changes after acute myocardial infarction are associated with an adverse short-term outcome in high risk cases, ${ }^{5}$ but the largest prognostic study of ambulatory ST segment monitoring for the detection of transient ischaemia after acute myocardial infarction did not show any significant value of ambulatory ST segment monitoring in identifying those more likely to have further acute myocardial infarction or sudden cardiac death over a mean two year follow up period. ${ }^{6}$ The authors reported a significantly increased risk of unstable angina and need for revascularisation (both subjective end points) in those with transient ischaemic changes, suggesting that those with ischaemia during their daily lives were more likely to develop symptoms in the medium-term future than those without.

Two studies have looked at the prognostic value of transient ST segment changes on ambulatory monitoring after CABG. ${ }^{1011}$ Egstrup suggested that such changes might help to identify those liable to have future cardiac events. Follow up time, however, was short (nine months), the study population small ( $\mathrm{n}=36$ ), and only one objective cardiac event was recorded..$^{10}$ Kennedy et al in a more recent and more extensive study have reported that transient, and almost exclusively silent ST segment changes are relatively frequent after surgery but that over a mean four year follow up period they did not help in identifying those more likely to have a 
subsequent cardiac event. ${ }^{11}$ They further concluded, on the basis of routine postoperative angiography, that such changes did not correlate with the degree of revascularisation. We have confirmed these prognostic findings at a follow up extending to 10 years after postoperative investigation, demonstrating no increase in events in patients with transient ST segment changes during daily life. Crea and colleagues had previously reported that ambulatory ST segment monitoring did not add further to the sensitivity of exercise tests in detecting incomplete revascularisation after CABG. ${ }^{17}$

The reasons for the lack of predictability from the findings of ambulatory ST segment monitoring are not clear. It is possible that some episodes of ST segment change after CABG might not in fact represent ischaemia. This is supported by Kennedy's observation that $99 \%$ of all such changes after surgery were symptom free, ${ }^{11}$ a figure far in excess of the expected frequency of silent ischaemia (about $70 \%$ ) in patients with stable angina. ${ }^{12}$ The combined effects of alteration in the electrocardiogram due to surgical manipulation and in many cases the tendency to tachycardia after withdrawal from $\beta$ blockers after operation may contribute to ST segment changes that do not necessarily represent ischaemia. ${ }^{13}$ It is possible that more rigid criteria for ischaemic change, such as $>2 \mathrm{~mm}$ ST segment depression might result in a greater specificity for the detection of ischaemia, although undoubtedly the sensitivity of such a test (already known to be $10 w^{18}$ ) would be further reduced. It is also well known that prognosis in stable coronary artery disease is very good, ${ }^{19}$ even in the presence of transient ischaemia, and this seems to be the case after CABG. Small areas of myocardium, which have not been completely revascularised, may become transiently ischaemic resulting in ST segment changes, but are unlikely to alter prognosis. It is also possible that in patients with transient ischaemia it is simply a marker of incomplete revascularisation and does not directly lead to the end points of coronary disease, in which case an increased incidence of further events would not be expected compared with patients without persisting ischaemia. Indeed little evidence to date shows a direct cause and effect correlation between ischaemia represented by $S T$ segment depression and acute myocardial infarction or sudden cardiac death. ${ }^{20-22}$

\section{LIMITATIONS OF THE STUDY}

Because this was not a planned prospective study there was a wide range of monitoring times after surgery. The mean time of investigation was 19 weeks, a sufficient length of time for sternal pain to have resolved, and for the patients to be fully ambulatory again. Follow up was taken as the time from postoperative monitoring, rather than from the time of surgery to events or to recent follow up. Although the study group is small, most had severe coronary disease and have had a considerable duration of follow up.

Although this group of patients was not consecutively studied for the purposes of this report, each of the two sub-groups were studied consecutively between 1982 and 1984 and between 1987 and 1988 as far as was possible depending on suitability, availability of monitoring equipment, and consent. We have attempted to exclude all patients who had acute ischaemic syndromes after CABG but before monitoring, but we have not excluded those few who underwent elective postoperative monitoring but had developed recurrent angina.

Twenty two per cent of patients were receiving antianginal medications at the time of ambulatory monitoring after CABG, some for coexistent hypertension. These patients were no less likely to have a subsequent cardiac event than those without treatment. A recent study by Yeung et al ${ }^{23}$ has shown that those patients receiving antianginal treatment that eradicated transient ischaemia during daily activities have a similar outlook to those patients with transient ischaemia but without treatment, suggesting that the reduction or eradication of ischaemia by medical means during daily life does not alter prognosis in terms of subsequent cardiac end points. Although patients on treatment in our study had less tendency to ischaemic change during daily activities, this did not translate into a better long-term outcome.

In conclusion ambulatory ST segment monitoring performed routinely after CABG detects transient (and predominantly silent) ST segment changes in a significant minority of patients, but such findings do not help to identify those patients more likely to have a subsequent major cardiac event up to 10 years after investigation. There is, therefore, little reason to perform this investigation in such patients. Reinvestigation should be reserved for patients who, after CABG are restricted by recurrent and restrictive symptoms of angina despite adequate medical treatment. The recommendation that patients with evidence of silent ischaemia on ambulatory monitoring after CABG should undergo reinvestigation and further intervention is not justified on the basis of the findings of this report.

1 Deanfield JE, Maseri A, Selwyn AP, et al. Myocardial ischaemia during daily life in patients with stable angina: its relation to symptoms and heart rate changes. Lance 1983;ii:753-8.

2 Mulcahy D, Keegan J, Crean P, et al. Silent myocardial ischaemia in chronic stable angina: a study of its frequency and characteristics in 150 patients. $\mathrm{Br}$ Heart $\mathcal{f}$ 1988;60:417-23.

3 Gottlieb SO, Weisfeldt ML, Ouyang P, Mellits ED Gerstanblith G. Silent ischaemia as a marker for early unfavourable outcomes in patients with unstable angina. unfavourable outcomes in patient

4 von Armin T, Gerbig HW, Krawietz, Hofling B. Prognostic implications of transient-predominantly Prognostic implications of transient-predominantly
silent - ischaemia in patients with unstable angina pecsilent - ischaemia in patients with

5 Gottlieb SO, Gottlieb SH, Achuff SC, et al. Silent ischaemia on Holter monitoring predicts mortality in ischaemia on Holter monitoring predicts mortality in Medical Association 1988;259:1030-5.

6 Tzivoni D, Gavish A, Zin D, et al. Prognostic significance of ischemic episodes in patients with previous myocardial infarction. Am $\mathcal{F}$ Cardiol 1988;62:661-4. 
7 Rocco MB, Nabel EG, Campbell S, et al. Prognostic importance of myocardial ischaemia detected by ambulatory monitoring in patients with stable

8 Deedwania PC, Carbajal EV. Silent ischemia during daily life is an independent predictor of mortality in stable angina. Circulation 1990;81:748-56.

9 Mulcahy D, Parameshwar J, Holdright D, et al. Value of ambulatory ST segment monitoring in patients with chronic stable angina: does measurement of the total ischaemic burden assist with management? Br Heart $f$ 1992;67:47-52.

10 Egstrup K. Asymptomatic myocardial ischaemia as a predictor of cardiac events after coronary artery bypass grafting for stable angina pectoris. Am 7 Cardiol 1988; 61:248-52.

11 Kennedy HL, Seiler SM, Sprague MK, et al. Relation of silent myocardial ischemia after coronary artery bypass grafting to angiographic completeness of revascularisation and long-term prognosis. Am $f$ Cardiol 1990;65: 14-22.

12 Quyyumi AA, Wright CA, Mockus LJ, Yacoub M, Fox KM. Effects of myocardial revascularisation in patients with effort angina and those with effort and nocturnal angina. Br Heart $\mathcal{F}$ 1985;54:557-61.

13 Mulcahy D, Keegan J, Phadke K, et al. Effects of coronary artery bypass surgery and angioplasty on the total ischaemic burden: a study of exercise testing and ambulatory ST segment monitoring. Am Heart $f$ 1992;123: 597-603.

14 Quyyumi AA, Mockus L, Wright C, Fox KM Morphology of ambulatory ST segment changes in patients with varying severity of coronary artery disease: investigation of the frequency of nocturnal ischaemia and coronary spasm. Br Heart $\mathcal{F}$ 1985;53:186-93.

15 Raby KE, Goldman L, Creager MA, et al. Correlation between pre-operative ambulatory ischemia and major cardiac events after peripheral vascular surgery. $N$ Engl $\mathcal{F}$ Med 1989;321:1296-300.

16 Raby KE, Goldman L, Cook EF, et al. Long-term prognosis of myocardial ischemia detected by holter monitoring in peripheral disease. Am $f$ Cardiol 1990;66: 1309-13.

17 Crea F, Kaski JC, Fragasso G, et al. Usefulness of Holter monitoring to improve the sensitivity of exercise testing in determining the degree of myocardial revascularisation after coronary artery bypass grafting for stable angina pectoris. Am $\mathcal{f}$ Cardiol 1987;60:40-3.

18 Mulcahy D, Keegan J, Sparrow J, Park A, Wright C, Fox KM. Ischemia in the ambulatory setting - the total ischemic burden: relation to exercise testing and investigative and therapeutic implications. $₹ \mathrm{Am}$ Coll Cardiol 1989;14:1166-72.

19 Brunelli C, Cristofani R, L'Abbate A. Long-term survival in medically treated patients with ischaemic heart disease and prognostic importance of clinical and electrocardiographic data. Eur Heart $\mathcal{f}$ 1989;10:292-303.

20 Assey ME, Walters GL, Hendrix GH, Carabello BA Usher BW, Spann JF. Incidence of acute myocardia infarction in patients with exercise induced silen myocardial ischemia. Am $\mathcal{f}$ Cardiol 1987;59:497-500.

21 Sharma B, Asinger R, Francis GS, Hodges M, Wyeth RP. Demonstration of painless exercise-induced myocardial ischemia in survivors of out-of-hospital ventricular fibrillation. Am $\mathcal{f}$ Cardiol 1987;59:740-5.

22 Mulcahy D, Keegan J, Cunningham D, et al. Circadian variation of total ischaemic burden and its alteration with anti-anginal agents. Lancet 1988;ii:755-9.

23 Yeung AC, Barry J, Orav J, Bonassin E, Raby KE, Selwyn AP. Effects of asymptomatic ischemia on long-term prognosis in chronic stable coronary disease. Circulation 1991;83:1598-604 